\title{
Variational Principles for Buckling of Microtubules Modeled as Nonlocal Orthotropic Shells
}

\author{
Sarp Adali \\ Discipline of Mechanical Engineering, University of KwaZulu-Natal, Durban 4041, South Africa \\ Correspondence should be addressed to Sarp Adali; adali@ukzn.ac.za
}

Received 15 April 2014; Revised 19 June 2014; Accepted 20 June 2014; Published 5 August 2014

Academic Editor: Chung-Min Liao

Copyright (c) 2014 Sarp Adali. This is an open access article distributed under the Creative Commons Attribution License, which permits unrestricted use, distribution, and reproduction in any medium, provided the original work is properly cited.

\begin{abstract}
A variational principle for microtubules subject to a buckling load is derived by semi-inverse method. The microtubule is modeled as an orthotropic shell with the constitutive equations based on nonlocal elastic theory and the effect of filament network taken into account as an elastic surrounding. Microtubules can carry large compressive forces by virtue of the mechanical coupling between the microtubules and the surrounding elastic filament network. The equations governing the buckling of the microtubule are given by a system of three partial differential equations. The problem studied in the present work involves the derivation of the variational formulation for microtubule buckling. The Rayleigh quotient for the buckling load as well as the natural and geometric boundary conditions of the problem is obtained from this variational formulation. It is observed that the boundary conditions are coupled as a result of nonlocal formulation. It is noted that the analytic solution of the buckling problem for microtubules is usually a difficult task. The variational formulation of the problem provides the basis for a number of approximate and numerical methods of solutions and furthermore variational principles can provide physical insight into the problem.
\end{abstract}

\section{Introduction}

Understanding the buckling characteristics of microtubules is of practical and theoretical importance since they perform a number of essential functions in living cells as discussed in [1-4]. In particular, they are the stiffest components of cytoskeleton and are instrumental in maintaining the shape of cells $[1,5]$. This is basically due to the fact that microtubules are able to support relatively large compressive loads as a result of coupling to the surrounding matrix. Since this function is of importance for cell mechanics and transmission of forces, the study of the buckling behavior of microtubules provides useful information on their biological functions. Consequently the buckling of microtubules has been studied employing increasingly more complicated continuum models which are often used to simulate their mechanical behavior and provide an effective tool to determine their load carrying capacity under compressive loads. The present study facilitates this investigation of the microtubule buckling problem by providing a variational setting which is the basis of a number of numerical and approximate solution methods.
Buckling of microtubules occurs for a number of reasons such as cell contraction or constrained microtubule polymerization at the cell periphery. To better understand this phenomenon, an effective approach is to use continuum models to represent a microtubule. These models include Euler-Bernoulli beam by Civalek and Demir [6], Timoshenko beam by Shi et al. [7], and cylindrical shells by Wang et al. [8] and $\mathrm{Gu}$ et al. [9]. The present study provides a variational formulation of the buckling of microtubules using an orthotropic shell model to represent their mechanical behavior. Variational principles form the basis of a number of computational and approximate methods of solution such as finite elements, Rayleigh-Ritz and Kantorovich. In particular Rayleigh quotient provides a useful expression to approximate the buckling load directly. As such the results presented can be used to obtain the approximate solutions for the buckling of microtubules as well as the variationally correct boundary conditions which are derived using the variational formulation of the problem.

Continuum modeling approach has been used effectively in other branches of biology and medicine [10], and their 
accuracy can be improved by implementing nonlocal constitutive relations for micro- and nanoscale phenomenon instead of classical local ones which relate the stress at a given point to the strain at the same point. As such local theories are of limited accuracy at the micro- and nanoscale since they neglect the small scale effects which can be substantial due to the atomic scale of the phenomenon. Recent examples of microtubule models based on the local elastic theory include [11-18] where Euler-Bernoulli and higher order shear deformable beams and cylindrical shells represented the microtubules. A review of the mechanical modeling of microtubules was given by Hawkins et al. [19] and a perspective on cell biomechanics by Ji and Bao [20].

In the present study the formulation is based on the nonlocal theory which accounts for the small scale effects and improves the accuracy. The nonlocal theory was developed in the early seventies by Eringen [21, 22] and recently applied to micro- and nanoscale structures. Nonlocal continuum models have been used in a number of studies to investigate the bending and vibration behavior of microtubules using nonlocal Euler-Bernoulli [6, 23] and Timoshenko beams [24]. There have been few studies on the buckling of microtubules based on a nonlocal theory. Nonlocal Timoshenko beam model was employed in [25-27] and nonlocal shell model in [1]. In a series of studies, Shen [28-30] used nonlocal shear deformable shell theory to study the buckling and postbuckling behavior of microtubules. Nonlocal problems also arise in other subject areas and have been studied using fractional calculus in a number studies [31, 32]. The models based on beam or isotropic shell theories neglect the directional dependence of the microtubule properties. The accuracy of a continuum model can be improved further by employing an orthotropic shell theory to take this directional dependence into account as discussed in [12, 33-35].

The objective of the present study is to derive a variational principle and Rayleigh quotient for the buckling load as well as the applicable natural and geometric boundary conditions for a microtubule subject to a compressive load. The particular model used in the study is a nonlocal orthotropic shell under a compressive load with the effect of filament network taken into account as an elastic surrounding. Moreover the pressure force on the microtubule exerted by the viscous cytosol is calculated using the Stokes flow theory [1]. The inclusion of these effects in the governing equations is important to model the phenomenon accurately since it is known that the microtubules can carry large compressive forces by virtue of the mechanical coupling between the microtubules and the surrounding elastic filament network as observed by Brangwynne et al. [36] and Das et al. [37]. Moreover, the microtubules are surrounded by the viscous cytosol in addition to the soft elastic filament network and the buckling causes the viscous flow of the cytosol [38]. These two processes result in an external stress field which improves the buckling characteristics of microtubules.

Variational formulations were employed in a number of studies involving microtubules. In particular, small scale formulations for the linear vibrations of microtubules were derived using the energy expression in [39, 40] and for nonlinear vibrations in [41]. Previous studies on variational principles involving nanoscale structures include multiwalled carbon nanotubes. In particular, variational principles were derived for nanotubes under buckling loads [42], for nanotubes undergoing linear vibrations [43], and for nanotubes undergoing nonlinear vibrations [44] using the nonlocal Euler-Bernoulli beam theories. Variational principles were also derived for nanotubes undergoing transverse vibrations using a nonlocal Timoshenko beam model in [45] and a strain-gradient cylindrical shell model in [46]. Apart from providing an insight into a physical problem, the variational formulations are often employed in the approximate and numerical solutions of the problems, in particular, in the presence of complicated boundary conditions [47]. Moreover natural boundary conditions can be easily derived from the variational formulation of the problem. In the present study variational formulation of a problem is derived by the semiinverse method developed by He [48-51] which was applied to several problems of mathematical physics to obtain the variational formulations for problems formulated in terms of differential equations [52-55]. Recently the semi-inverse method was applied to the heat conduction equation in [56] to obtain a constrained variational principle. The equivalence of this formulation to the one obtained by He and Lee [57] has been shown in $[58,59]$. A recent application of the semi-inverse method involves the derivation of variational principles for partial differential equations modeling water transport in porous media [60].

\section{Equations Based on Nonlocal Elastic Theory}

In the present study the microtubule is modeled as an orthotropic cylindrical shell of length $L$, radius $R$, and wall thickness $h$ and surrounded by a viscoelastic medium (cytoplasm). It is subject to an axial compressive load $N$ as shown in Figure 1.

The microtubule has the Young's moduli $E_{1}$ and $E_{2}$ along the axial and circumferential directions, shear modulus $G$ and Poisson's ratios $\mu_{1}$ and $\mu_{2}$ along the circumferential and axial directions. Dimensionless longitudinal direction is denoted by $x=X / R$ and the circumferential direction by $\theta$ as in Figure 1. For an orthotropic shell, the constitutive relations based on nonlocal elasticity theory are given by (see [1])

$$
\begin{aligned}
\sigma_{x}-\frac{\left(e a_{0}\right)^{2}}{R^{2}} \nabla^{2} \sigma_{x} & =\frac{E_{1}}{1-\mu_{1} \mu_{2}} \varepsilon_{x}+\frac{E_{2} \mu_{2}}{1-\mu_{1} \mu_{2}} \varepsilon_{\theta}, \\
\sigma_{\theta}-\frac{\left(e a_{0}\right)^{2}}{R^{2}} \nabla^{2} \sigma_{\theta} & =\frac{E_{2}}{1-\mu_{1} \mu_{2}} \varepsilon_{\theta}+\frac{E_{1} \mu_{1}}{1-\mu_{1} \mu_{2}} \varepsilon_{x}, \\
\tau_{x \theta}-\frac{\left(e a_{0}\right)^{2}}{R^{2}} \nabla^{2} \tau_{x \theta} & =G \varepsilon_{x \theta},
\end{aligned}
$$

where $\nabla^{2}=\left(\partial^{2} / \partial x^{2}\right)+\left(\partial^{2} / \partial \theta^{2}\right)$ is the Laplace operator, $\sigma_{x}, \sigma_{\theta}$, and $\tau_{x \theta}$ are stress components, and $\varepsilon_{x}, \varepsilon_{\theta}$, and $\varepsilon_{x \theta}$ are the normal and shear strains. In (1), $e a_{0}$ is the small scale parameter reflecting the nanoscale of the phenomenon and has to be experimentally determined. The differential equations governing the buckling of the microtubules are 


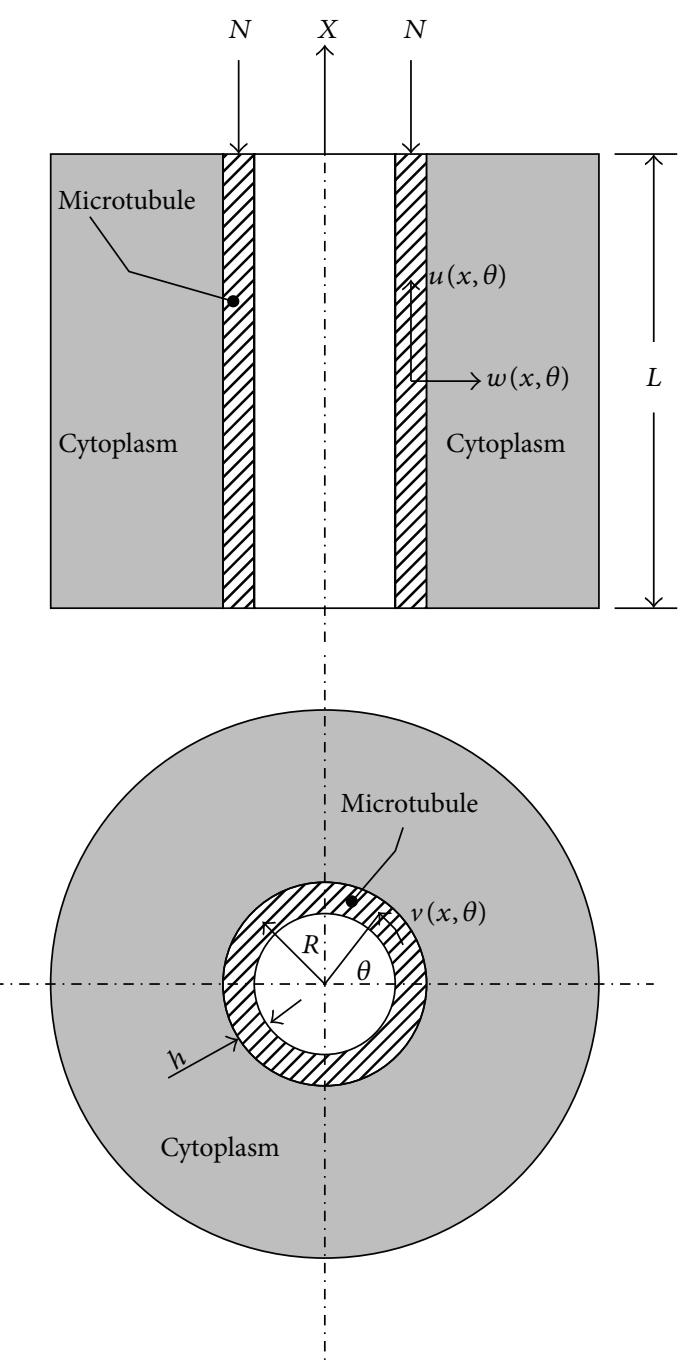

Figure 1: Microtubule under a compressive load and its surrounding.

given in [1] based on the nonlocal constitutive relation (1). The differential equation formulation of the problem is expressed as a system of partial differential equations given by

$$
\begin{aligned}
& D_{1}(u, v, w) \equiv L_{1}(u)+M_{1}(v, w)=0, \\
& D_{2}(u, v, w) \equiv L_{2}(v)+M_{2}(u, w)=0, \\
& D_{3}(u, v, w) \equiv L_{3}(w)+M_{3}(u, v)=0,
\end{aligned}
$$

where $u(x, \theta), v(x, \theta)$, and $w(x, \theta)$ are the displacement components in the axial, circumferential, and radial directions, respectively, as shown in Figure 1. The differential operators $L_{i}$ and $M_{i}$ are defined as

$$
\begin{gathered}
L_{1}(u)=k_{2}\left(1+c^{2}\right) u_{\theta \theta}+u_{x x}+\frac{N}{K} \mathscr{L}\left(u_{x x}\right), \\
M_{1}(v, w)=\left(k_{2}+\mu_{1}\right) v_{x \theta}+\mu_{1} w_{x}-c^{2} w_{x x x}+c^{2} k_{2} w_{x \theta \theta}, \\
L_{2}(v)=k_{2}\left(1+3 c^{2}\right) v_{x x}+k_{1} v_{\theta \theta}+\frac{N}{K} \mathscr{L}\left(v_{x x}\right),
\end{gathered}
$$

$$
\begin{aligned}
M_{2}(u, w)= & \left(k_{2}+\mu_{1}\right) u_{x \theta}+k_{1} w_{\theta}-c^{2}\left(3 k_{2}+\mu_{1}\right) w_{x x \theta}, \\
L_{3}(w)= & -\left(1+c^{2}\right) k_{1} w-2 c^{2} k_{1} w_{\theta \theta} \\
& -c^{2}\left(w_{x x x x}+k_{1} w_{\theta \theta \theta \theta}+\left(4 k_{2}+2 \mu_{1}\right) w_{x x \theta \theta}\right) \\
& +\frac{c R}{K} \mathscr{L}(w)+\frac{N}{K} \mathscr{L}\left(w_{x x}\right)+\frac{R}{K} \mathscr{L}\left(P_{Y Y}\right), \\
& M_{3}(u, v)=-\mu_{1} u_{x}+c^{2}\left(u_{x x x}-k_{2} u_{x \theta \theta}\right) \\
& -k_{1} v_{\theta}+c^{2}\left(3 k_{2}+\mu_{1}\right) v_{x x \theta},
\end{aligned}
$$

where the differential operator $\mathscr{L}(\cdot)$ is defined as $\mathscr{L}(\cdot)=$ $\eta^{2} \nabla^{2}-1$, the subscripts $x, \theta$ denote differentiation with respect to that variable, and the dimensionless small scale parameter $\eta$ is given by $\eta=e a_{0} / R$. The symbols in (3)-(8) are defined as

$$
\begin{aligned}
k_{1} & =\frac{E_{1}}{E_{2}}, \\
k_{2} & =\frac{G\left(1-\mu_{1} \mu_{2}\right)}{E_{1}}, \\
c^{2} & =\frac{h_{0}^{3}}{12 R^{2} h}, \\
K & =\frac{E_{1} h}{\left(1-\mu_{1} \mu_{2}\right)},
\end{aligned}
$$

where $h_{0}$ is the effective thickness for bending. The symbol $\varsigma$ appearing in (7) is the elastic constraint from the filaments network and is given by $\varsigma=2.7 E_{c}$ where $E_{c}$ is the elastic modulus of the surrounding viscoelastic medium. The radial pressure $P_{Y Y}$ exerted by the motion of cytosol in (7) is computed from the dynamic equations of cytosol given in [1].

\section{Variational Formulation}

Following the semi-inverse method, we construct a variational trial-functional $V(u, v, w)$ as follows:

$$
\begin{aligned}
V(u, v, w)= & V_{1}(u)+V_{2}(v)+V_{3}(w) \\
& +\int_{0}^{2 \pi} \int_{0}^{l} F(u, v, w) d x d \theta,
\end{aligned}
$$

where $l=L / R, x \in[0, L / R]$ and the functionals $V_{1}(u), V_{2}(v)$, $V_{3}(w)$ are given by

$$
\begin{aligned}
& V_{1}(u)=\frac{1}{2} \int_{0}^{2 \pi} \int_{0}^{l}[-k_{2}\left(1+c^{2}\right) u_{\theta}^{2}-u_{x}^{2} \\
&\left.+\frac{N}{K}\left(\eta^{2}\left(u_{x x}^{2}+u_{x \theta}^{2}\right)+u_{x}^{2}\right)\right] d x d \theta, \\
& V_{2}(v)=\frac{1}{2} \int_{0}^{2 \pi} \int_{0}^{l}\left[-k_{2}\left(1+3 c^{2}\right) v_{x}^{2}-k_{1} v_{\theta}^{2}\right. \\
&\left.+\frac{N}{K}\left(\eta^{2}\left(v_{x x}^{2}+v_{x \theta}^{2}\right)+v_{x}^{2}\right)\right] d x d \theta
\end{aligned}
$$




$$
\begin{aligned}
& V_{3}(w) \\
& \begin{aligned}
& \frac{1}{2} \int_{0}^{2 \pi} \int_{0}^{l}[-\left(1+c^{2}\right) k_{1} w^{2}+2 c^{2} k_{1} w_{\theta}^{2} \\
&-c^{2}\left(w_{x x}^{2}+k_{1} w_{\theta \theta}^{2}\right) \\
&-\left.\left(4 k_{2}+2 \mu_{1}\right) w_{x \theta}^{2}\right] d x d \theta \ldots \\
&+\frac{1}{2} \int_{0}^{2 \pi} \int_{0}^{l}\left[-\frac{\varsigma R}{K}\left(\eta^{2}\left(w_{x}^{2}+w_{\theta}^{2}\right)+w^{2}\right)+\frac{N}{K}\right. \\
& \times\left(\eta^{2}\left(w_{x x}^{2}+w_{x \theta}^{2}\right)+w_{x}^{2}\right) \\
&\left.+\frac{2 R}{K} \mathscr{L}\left(P_{Y Y}\right) w\right] d x d \theta .
\end{aligned}
\end{aligned}
$$

In (10), $F(u, v, w)$ is an unknown function to be determined such that the Euler-Lagrange equations of the variational functional (10) correspond to the differential equation (2). This establishes the direct relation between the variational formulation and the governing equations in the sense that differential equation (2) can be obtained from the derived variational principle using the Euler-Lagrange equations. It is noted that the choice of the trial functionals defined by (10)-(11) is not unique. The review article by $\mathrm{He}$ [61] provides a systematic treatment on the use of semi-inverse method for the derivation of variational principles and the selection of trial functionals as well as on variational methods for the solution of linear and nonlinear problems.

It is noted that the Euler-Lagrange equations of the variational functional $V(u, v, w)$ are

$$
\begin{gathered}
L_{1}(u)+\frac{\delta F}{\delta u}=0, \\
L_{2}(v)+\frac{\delta F}{\delta v}=0, \\
L_{3}(w)+\frac{\delta F}{\delta w}=0 .
\end{gathered}
$$

Thus the functionals $V_{1}(u), V_{2}(v)$, and $V_{3}(w)$ are the variational functionals for the differential operators $L_{1}(u), L_{2}(v)$, and $L_{3}(w)$, respectively. In (12), the variational derivative $\delta F / \delta u$ is defined as

$$
\begin{aligned}
\frac{\delta F}{\delta u}= & \frac{\partial F}{\partial u}-\frac{\partial}{\partial x}\left(\frac{\partial F}{\partial u_{x}}\right)-\frac{\partial}{\partial \theta}\left(\frac{\partial F}{\partial u_{\theta}}\right)+\frac{\partial^{2}}{\partial x^{2}}\left(\frac{\partial F}{\partial u_{x x}}\right) \\
& +\frac{\partial^{2}}{\partial x \partial \theta}\left(\frac{\partial F}{\partial u_{x \theta}}\right)+\frac{\partial^{2}}{\partial \theta^{2}}\left(\frac{\partial F}{\partial u_{\theta \theta}}\right) \\
& -\frac{\partial^{3}}{\partial x^{3}}\left(\frac{\partial F}{\partial u_{x x x}}\right) \cdots
\end{aligned}
$$

Comparing (12) with (2), we observe that the following equations have to be satisfied for Euler-Lagrange equations of $V(u, v, w)$ to represent the governing equation (2), namely,

$$
\begin{aligned}
\frac{\delta F}{\delta u}= & M_{1}(v, w) \\
= & \left(k_{2}+\mu_{1}\right) v_{x \theta}+\mu_{1} w_{x} \\
& -c^{2} w_{x x x}+c^{2} k_{2} w_{x \theta \theta},
\end{aligned}
$$

$$
\begin{aligned}
\frac{\delta F}{\delta v}= & M_{2}(u, w)=\left(k_{2}+\mu_{1}\right) u_{x \theta}+k_{1} w_{\theta} \\
& -c^{2}\left(3 k_{2}+\mu_{1}\right) w_{x x \theta}, \\
\frac{\delta F}{\delta w}= & M_{3}(u, v) \\
= & -\mu_{1} u_{x}+c^{2}\left(u_{x x x}-k_{2} u_{x \theta \theta}\right) \\
& -k_{1} v_{\theta}+c^{2}\left(3 k_{2}+\mu_{1}\right) v_{x x \theta} .
\end{aligned}
$$

The function $F(u, v, w)$ has to be determined such that (14)-(16) are satisfied. For this purpose we first determine $F(u, v, w)$ satisfying (14) to obtain

$$
\begin{aligned}
F(u, v, w)= & -\left(k_{2}+\mu_{1}\right) v_{x} u_{\theta}+\mu_{1} w_{x} u+c^{2} w_{x x} u_{x} \\
& +c^{2} k_{2} w_{x} u_{\theta \theta}+\Phi(v, w),
\end{aligned}
$$

where $\Phi(v, w)$ is an unknown function of $v$ and $w$. Next we compute $\delta F / \delta v$ from (17), namely,

$$
\frac{\delta F}{\delta v}=\left(k_{2}+\mu_{1}\right) u_{x \theta}+\frac{\delta \Phi(v, w)}{\delta v}
$$

which should satisfy (15). From (15) and (18) it follows that

$$
\frac{\delta \Phi(v, w)}{\delta v}=k_{1} w_{\theta}-c^{2}\left(3 k_{2}+\mu_{1}\right) w_{x x \theta}
$$

The expression for $\Phi(v, w)$ satisfying (19) is determined as

$$
\Phi(v, w)=-k_{1} w v_{\theta}+c^{2}\left(3 k_{2}+\mu_{1}\right) w_{x x} v_{\theta}
$$

Thus from (17) and (20), $F(u, v, w)$ is obtained as

$$
\begin{aligned}
F(u, v, w)= & -\left(k_{2}+\mu_{1}\right) v_{x} u_{\theta}+\mu_{1} w_{x} u+c^{2} w_{x x} u_{x} \\
& +c^{2} k_{2} w_{x} u_{\theta \theta}-k_{1} w v_{\theta} \\
& +c^{2}\left(3 k_{2}+\mu_{1}\right) w_{x x} v_{\theta} .
\end{aligned}
$$

Finally we note that $\partial F / \partial w$ satisfies (16). Thus the function $F(u, v, w)$ given by $(21)$ satisfies the Euler-Lagrange equations (14)-(16) as required. Now the variational functional can be expressed as

$$
V(u, v, w)=V_{1}(u)+V_{2}(v)+V_{3}(w)+V_{4}(u, v, w),
$$

where

$$
\begin{aligned}
& V_{4}(u, v, w) \\
& =\int_{0}^{2 \pi} \int_{0}^{l} F(u, v, w) d x d \theta \\
& =\int_{0}^{2 \pi} \int_{0}^{l}\left(-\left(k_{2}+\mu_{1}\right) v_{x} u_{\theta}+\mu_{1} w_{x} u+c^{2} w_{x x} u_{x}\right. \\
& +c^{2} k_{2} w_{x} u_{\theta \theta}-k_{1} w v_{\theta} \\
& \left.+c^{2}\left(3 k_{2}+\mu_{1}\right) w_{x x} v_{\theta}\right) d x d \theta
\end{aligned}
$$

and $V_{1}(u), V_{2}(v)$, and $V_{3}(w)$ are given by (11). To verify the validity of the variational formulation (22), one has to show that the Euler-Lagrange equations of $V(u, v, w)$ yield the governing (2). The fact that this is indeed the case can be shown easily. 


\section{Rayleigh Quotient}

The Raleigh quotient is obtained for the buckling load $N$ by noting that

$$
\begin{gathered}
V_{1}(u)=-R_{1}(u)+N S(u), \\
V_{2}(v)=-R_{2}(v)+N S(v), \\
V_{3}(w)=-R_{3}(w)+N S(w),
\end{gathered}
$$

where

$$
\begin{gathered}
R_{1}(u)=\frac{1}{2} \int_{0}^{2 \pi} \int_{0}^{l}\left(k_{2}\left(1+c^{2}\right) u_{\theta}^{2}+u_{x}^{2}\right) d x d \theta \\
R_{2}(v)=\frac{1}{2} \int_{0}^{2 \pi} \int_{0}^{l}\left(k_{2}\left(1+3 c^{2}\right) v_{x}^{2}+k_{1} v_{\theta}^{2}\right) d x d \theta \\
R_{3}(w) \\
=\frac{1}{2} \int_{0}^{2 \pi} \int_{0}^{l}\left(\left(1+c^{2}\right) k_{1} w^{2}-2 c^{2} k_{1} w_{\theta}^{2}\right. \\
+c^{2}\left(w_{x x}^{2}+k_{1} w_{\theta \theta}^{2}\right) \\
+\frac{1}{2} \int_{0}^{2 \pi} \int_{0}^{l}\left(\frac{\varsigma R}{K}\left(\eta^{2}\left(w_{x}^{2}+w_{\theta}^{2}\right)+w^{2}\right)\right. \\
S(y)=\frac{1}{2 K} \int_{0}^{2 \pi} \int_{0}^{l}\left(\eta^{2}\left(y_{x x}^{2}+y_{x \theta}^{2}\right)+y_{x}^{2}\right) d x d \theta
\end{gathered}
$$

Thus from (22) and (24), it follows that

$$
\begin{aligned}
V(u, v, w)= & -\left(R_{1}(u)+R_{2}(v)+R_{3}(u)\right) \\
& +V_{4}(u, v, w) \\
& +N(S(u)+S(v)+S(w))
\end{aligned}
$$

and the Rayleigh quotient can be expressed as

$$
N=\min _{u, v, w} \frac{R_{1}(u)+R_{2}(v)+R_{3}(w)-V_{4}(u, v, w)}{S(u)+S(v)+S(w)} .
$$

\section{Natural and Geometric Boundary Conditions}

It is noted that the displacements are equal at the end points $\theta=0$ and $\theta=2 \pi$; that is,

$$
\begin{gathered}
u(x, 0)=u(x, 2 \pi), \\
v(x, 0)=v(x, 2 \pi), \\
w(x, 0)=w(x, 2 \pi) \\
\text { for } x \in[0, l] .
\end{gathered}
$$

The first variations of $V(u, v, w)$ with respect to $\delta u, \delta v$, and $\delta w$, denoted by $\delta_{u} V, \delta_{v} V$, and $\delta_{w} V$, respectively, can be obtained by integration by parts and using (28). We first obtain the variations of $V_{1}(u)$ and $V_{4}(u, v, w)$ with respect to $\delta u$ which are given by

$$
\begin{aligned}
& \delta_{u} V_{1}(u) \\
&= \int_{0}^{2 \pi} \int_{0}^{l} L_{1}(u) \delta u d x d \theta+B_{1}\left(u, \delta u, \delta u_{x}\right), \\
& \delta_{u} V_{4}(u, v, w) \\
&=\int_{0}^{2 \pi} \int_{0}^{l} M_{1}(v, w) \delta u d x d \theta+B_{2}(w, \delta u),
\end{aligned}
$$

where

$$
\begin{gathered}
B_{1}\left(u, \delta u, \delta u_{x}\right) \\
=\int_{0}^{2 \pi}\left[\left(-u_{x}-\frac{N}{K}\left(\eta^{2}\left(u_{x x x}+u_{x \theta \theta}\right)-u_{x}\right)\right) \delta u\right. \\
\left.+\frac{N}{K} \eta^{2} u_{x x} \delta u_{x}\right]_{x=0}^{x=l} d \theta \\
B_{2}(w, \delta u)=\int_{0}^{2 \pi}\left[c^{2} w_{x x} \delta u\right]_{x=0}^{x=l} d \theta .
\end{gathered}
$$

Similarly

$$
\begin{aligned}
\delta_{v} V_{2}(v)= & \int_{0}^{2 \pi} \int_{0}^{l} L_{2}(v) \delta v d x d \theta+B_{3}\left(v, \delta v, \delta v_{x}\right), \\
\delta_{v} V_{4}(u, v, w)= & \int_{0}^{2 \pi} \int_{0}^{l} M_{2}(u, w) \delta v d x d \theta \\
& +B_{4}(u, \delta v),
\end{aligned}
$$

where

$$
\begin{gathered}
B_{3}\left(v, \delta v, \delta v_{x}\right) \\
=\int_{0}^{2 \pi}\left[\left(-k_{2}\left(1+3 c^{2}\right) v_{x}-\frac{N}{K}\left(\eta^{2}\left(v_{x x x}+v_{x \theta \theta}\right)-v_{x}\right)\right) \delta v\right. \\
\left.+\frac{N}{K} \eta^{2} v_{x x} \delta v_{x}\right]_{x=0}^{x=l} d \theta \\
B_{4}(u, \delta v)=\int_{0}^{2 \pi}\left[-\left(k_{2}+\mu_{1}\right) u_{\theta}\right]_{x=0}^{x=l} \delta v d \theta .
\end{gathered}
$$

Finally we obtain $\delta_{w} V_{3}(w)$ and $\delta_{w} V_{4}(u, v, w)$, namely,

$$
\begin{aligned}
\delta_{w} V_{3}(w) & \\
= & \int_{0}^{2 \pi} \int_{0}^{l} L_{3}(w) \delta w d x d \theta+B_{5}\left(w, \delta w, \delta w_{x}\right) \\
& +B_{6}\left(w, \delta w, \delta w_{x}\right),
\end{aligned}
$$




$$
\begin{aligned}
\delta_{w} V_{4}(u, v, w) \\
=\int_{0}^{2 \pi} \int_{0}^{l} M_{3}(u, v) \delta w d x d \theta+B_{7}\left(u, v, \delta w, \delta w_{x}\right) \\
\quad+B_{7}\left(u, v, \delta w, \delta w_{x}\right)
\end{aligned}
$$

where

$$
\begin{aligned}
& B_{5}\left(w, \delta w, \delta w_{x}\right) \\
& =\int_{0}^{2 \pi}\left[c^{2}\left(w_{x x x}+\left(4 k_{2}+2 \mu_{1}\right) w_{x \theta \theta}\right) \delta w\right. \\
& \left.\quad-c^{2} w_{x x} \delta w_{x}\right]_{x=0}^{x=l} d \theta \\
& B_{6}\left(w, \delta w, \delta w_{x}\right) \\
& =\int_{0}^{2 \pi}\left[\left(-\frac{\varsigma R}{K} \eta^{2} w_{x}-\frac{N}{K}\left(\eta^{2}\left(w_{x x x}+w_{x \theta \theta}\right)-w_{x}\right)\right) \delta w\right. \\
& \left.\quad+\frac{N}{K} \eta^{2} w_{x x} \delta w_{x}\right]_{x=0}^{x=l} d \theta, \\
& B_{7}\left(u, v, \delta w, \delta w_{x}\right) \\
& =\int_{0}^{2 \pi}\left[\left(\mu_{1} u+c^{2}\left(k_{2} u_{\theta \theta}-u_{x x}\right)-c^{2}\left(3 k_{2}+\mu_{1}\right) v_{x \theta}\right) \delta w\right. \\
& \left.+\left(c^{2}\left(3 k_{2}+\mu_{1}\right) v_{\theta}+c^{2} u_{x}\right) \delta w_{x}\right]_{x=0}^{x=l} d \theta .
\end{aligned}
$$

Since the first variations of the functional $V(u, v, w)$ are zero, that is,

$$
\delta_{u} V(u, v, w)=\delta_{v} V(u, v, w)=\delta_{w} V(u, v, w)=0
$$

by the fundamental lemma of the calculus of variations, we have

$$
\begin{aligned}
& B_{1}\left(u, \delta u, \delta u_{x}\right)+B_{2}(w, \delta u)+B_{3}\left(v, \delta v, \delta v_{x}\right) \\
& \quad+B_{4}(u, \delta v)+B_{5}\left(w, \delta w, \delta w_{x}\right) \\
& \quad+B_{6}\left(w, \delta w, \delta w_{x}\right)+B_{7}\left(u, v, \delta w, \delta w_{x}\right)=0
\end{aligned}
$$

which yields the boundary conditions. We first note that (36) can be written as

$$
\sum_{i=1}^{5} b_{i}=0
$$

where

$$
\begin{gathered}
b_{1}\left(u, w, \delta u, \delta u_{x}\right) \\
=\int_{0}^{2 \pi}\left[\left(-u_{x}-\frac{N}{K}\left(\eta^{2}\left(u_{x x x}+u_{x \theta \theta}\right)-u_{x}\right)+c^{2} w_{x x}\right) \delta u\right. \\
\left.+\frac{N}{K} \eta^{2} u_{x x} \delta u_{x}\right]_{x=0}^{x=l} d \theta
\end{gathered}
$$

$b_{2}\left(u, v, w, \delta v, \delta v_{x}\right)$

$$
\begin{gathered}
\int_{0}^{2 \pi}\left[\left(-\left(k_{2}+\mu_{1}\right) u_{\theta}-k_{2}\left(1+3 c^{2}\right) v_{x}\right.\right. \\
-\frac{N}{K}\left(\eta^{2}\left(v_{x x x}+v_{x \theta \theta}\right)-v_{x}\right) \\
\left.\left.-c^{2}\left(3 k_{2}+\mu_{1}\right) w_{x \theta}\right) \delta v\right]_{x=0}^{x=l} d \theta \\
+\int_{0}^{2 \pi}\left[\frac{N}{K} \eta^{2} v_{x x} \delta v_{x}\right]_{x=0}^{x=l} d \theta,
\end{gathered}
$$

$b_{3}(w, \delta w)$

$$
\begin{aligned}
\int_{0}^{2 \pi}\left[\left(c^{2}\left(w_{x x x}+\left(4 k_{2}+2 \mu_{1}\right) w_{x \theta \theta}\right)-\frac{\varsigma R}{K} \eta^{2} w_{x}\right.\right. \\
\left.\left.-\frac{N}{K}\left(\eta^{2}\left(w_{x x x}+w_{x \theta \theta}\right)-w_{x}\right)\right) \delta w\right]_{x=0}^{x=l} d \theta,
\end{aligned}
$$

$b_{4}(u, v, \delta w)$

$$
\begin{aligned}
=\int_{0}^{2 \pi}\left[\left(\mu_{1} u+c^{2}\left(k_{2} u_{\theta \theta}-u_{x x}\right)\right.\right. \\
\left.\left.\quad-c^{2}\left(3 k_{2}+\mu_{1}\right) v_{x \theta}\right) \delta w\right]_{x=0}^{x=l} d \theta,
\end{aligned}
$$

$b_{5}\left(u, v, w, \delta w_{x}\right)$

$$
\begin{gathered}
\int_{0}^{2 \pi}\left[\left(-c^{2} w_{x x}+\frac{N}{K} \eta^{2} w_{x x}+c^{2}\left(3 k_{2}+\mu_{1}\right) v_{\theta}\right.\right. \\
\left.\left.+c^{2} u_{x}\right) \delta w_{x}\right]_{x=0}^{x=l} d \theta .
\end{gathered}
$$

From (37)-(38), the natural and geometric boundary conditions are obtained at $x=0$ and $x=l$ as

$$
\begin{gathered}
u_{x}+\frac{N}{K}\left(\eta^{2}\left(u_{x x x}+u_{x \theta \theta}\right)-u_{x}\right)-c^{2} w_{x x}=0 \quad \text { or } u=0, \\
u_{x x}=0 \quad \text { or } \quad u_{x}=0 \\
\left(k_{2}+\mu_{1}\right) u_{\theta}+k_{2}\left(1+3 c^{2}\right) v_{x} \\
+\frac{N}{K}\left(\eta^{2}\left(v_{x x x}+v_{x \theta \theta}\right)-v_{x}\right) \\
+c^{2}\left(3 k_{2}+\mu_{1}\right) w_{x \theta}=0 \quad \text { or } \quad v=0, \\
v_{x x}^{2}\left(w_{x x x}+\left(4 k_{2}+2 \mu_{1}\right) w_{x \theta \theta}\right)-\frac{\varsigma R}{K} \eta^{2} w_{x} \\
-\frac{N}{K}\left(\eta^{2}\left(w_{x x x}+w_{x \theta \theta}\right)-w_{x}\right) \cdots+\mu_{1} u \\
+c^{2}\left(k_{2} u_{\theta \theta}-u_{x x}\right) \\
-c^{2}\left(3 k_{2}+\mu_{1}\right) v_{x \theta}=0 \quad \text { or } \quad w=0 \\
-c^{2} w_{x x}+\frac{N}{K} \eta^{2} w_{x x}+c^{2}\left(3 k_{2}+\mu_{1}\right) v_{\theta} \\
+c^{2} u_{x}=0 \quad \text { or } \quad w_{x}=0 .
\end{gathered}
$$




\section{Conclusions}

The variational formulation for the buckling of a microtubule was given using a nonlocal continuum theory whereby the microtubule was modeled as an orthotropic shell. The continuum model of the microtubule takes the effects of the surrounding filament network and the viscous cytosol into account as well as its orthotropic properties. Methods of calculus of variations were employed in the derivation of the variational formulation and in particular the semiinverse approach was used to identify suitable variational integrals. The buckling load was expressed in the form of a Rayleigh quotient which confirms that small scale effects lower the buckling load as has been observed in a number of studies $[1,26,28]$. The natural and geometric boundary conditions were derived using the formulations developed. The variational principles presented here form the basis of several approximate and numerical methods of solution and facilitate the implementation of complicated boundary conditions, in particular, the natural boundary conditions.

\section{Conflict of Interests}

The author declares that there is no conflict of interests regarding the publication of this paper.

\section{Acknowledgments}

The research reported in this paper was supported by research grants from the University of KwaZulu-Natal (UKZN) and from National Research Foundation (NRF) of South Africa. The author gratefully acknowledges the support provided by UKZN and NRF.

\section{References}

[1] Y. Gao and L. An, "A nonlocal elastic anisotropic shell model for microtubule buckling behaviors in cytoplasm," Physica E: Low-Dimensional Systems and Nanostructures, vol. 42, no. 9, pp. 2406-2415, 2010

[2] M. Taj and J.-Q. Zhang, "Buckling of embedded microtubules in elastic medium," Applied Mathematics and Mechanics, vol. 32, no. 3, pp. 293-300, 2011.

[3] P. Xiang and K. M. Liew, "Predicting buckling behavior of microtubules based on an atomistic-continuum model," International Journal of Solids and Structures, vol. 48, no. 11-12, pp. 1730-1737, 2011.

[4] P. Xiang and K. M. Liew, "A computational framework for transverse compression of microtubules based on a higher-order Cauchy-Born rule," Computer Methods in Applied Mechanics and Engineering, vol. 254, pp. 14-30, 2013.

[5] Y. Ujihara, M. Nakamura, H. Miyazaki, and S. Wada, "Segmentation and morphometric analysis of cells from fluorescence microscopy images of cytoskeletons," Computational and Mathematical Methods in Medicine, vol. 2013, Article ID 381356, 11 pages, 2013.

[6] Ö. Civalek and Ç. Demir, "Bending analysis of microtubules using nonlocal Euler-Bernoulli beam theory," Applied Mathematical Modelling, vol. 35, no. 5, pp. 2053-2067, 2011.
[7] Y. J. Shi, W. L. Guo, and C. Q. Ru, "Relevance of Timoshenkobeam model to microtubules of low shear modulus," Physica E: Low-Dimensional Systems and Nanostructures, vol. 41, no. 2, pp. 213-219, 2008.

[8] C. Y. Wang, C. Q. Ru, and A. Mioduchowski, "Orthotropic elastic shell model for buckling of microtubules," Physical Review E. Statistical, Nonlinear, and Soft Matter Physics, vol. 74, no. 5, Article ID 052901, 2006.

[9] B. Gu, Y.-W. Mai, and C. Q. Ru, "Mechanics of microtubules modeled as orthotropic elastic shells with transverse shearing," Acta Mechanica, vol. 207, no. 3-4, pp. 195-209, 2009.

[10] T. Heidlauf and O. Röhrle, "Modeling the chemoelectromechanical behavior of skeletal muscle using the parallel opensource software library openCMISS," Computational and Mathematical Methods in Medicine, vol. 2013, Article ID 517287, 14 pages, 2013.

[11] E. Ghavanloo, F. Daneshmand, and M. Amabili, "Vibration analysis of a single microtubule surrounded by cytoplasm," Physica E: Low-Dimensional Systems and Nanostructures, vol. 43, no. 1, pp. 192-198, 2010.

[12] T. Li, "A mechanics model of microtubule buckling in living cells," Journal of Biomechanics, vol. 41, no. 8, pp. 1722-1729, 2008.

[13] X. S. Qian, J. Q. Zhang, and C. Q. Ru, "Wave propagation in orthotropic microtubules," Journal of Applied Physics, vol. 101, no. 8, Article ID 084702, 2007.

[14] A. Tounsi, H. Heireche, H. Benhassaini, and M. Missouri, "Vibration and length-dependent flexural rigidity of protein microtubules using higher order shear deformation theory," Journal of Theoretical Biology, vol. 266, no. 2, pp. 250-255, 2010.

[15] C. Y. Wang, C. Q. Ru, and A. Mioduchowski, "Vibration of microtubules as orthotropic elastic shells," Physica E: LowDimensional Systems and Nanostructures, vol. 35, no. 1, pp. 48$56,2006$.

[16] C. Y. Wang, C. F. Li, and S. Adhikari, "Dynamic behaviors of microtubules in cytosol," Journal of Biomechanics, vol. 42, no. 9, pp. 1270-1274, 2009.

[17] C. Y. Wang and L. C. Zhang, "Circumferential vibration of microtubules with long axial wavelength," Journal of Biomechanics, vol. 41, no. 9, pp. 1892-1896, 2008.

[18] L. Yi, T. Chang, and C. Q. Ru, "Buckling of microtubules under bending and torsion," Journal of Applied Physics, vol. 103, no. 10, Article ID 103516, 2008.

[19] T. Hawkins, M. Mirigian, M. S. Yasar, and J. L. Ross, "Mechanics of microtubules," Journal of Biomechanics, vol. 43, no. 1, pp. 2330, 2010.

[20] B. Ji and G. Bao, "Cell and molecular biomechanics: perspectives and challenges," Acta Mechanica Solida Sinica, vol. 24, no. 1, pp. 27-51, 2011.

[21] A. C. Eringen, "Linear theory of nonlocal elasticity and dispersion of plane waves," International Journal of Engineering Science, vol. 10, pp. 425-435, 1972.

[22] A. C. Eringen, "On differential equations of nonlocal elasticity and solutions of screw dislocation and surface waves," Journal of Applied Physics, vol. 54, no. 9, pp. 4703-4710, 1983.

[23] Ö. Civalek, Ç. Demir, and B. Akgöz, "Free vibration and bending analyses of Cantilever microtubules based on nonlocal continuum model," Mathematical \& Computational Applications, vol. 15, no. 2, pp. 289-298, 2010.

[24] H. Heireche, A. Tounsi, H. Benhassaini et al., "Nonlocal elasticity effect on vibration characteristics of protein microtubules," 
Physica E: Low-Dimensional Systems and Nanostructures, vol. 42, no. 9, pp. 2375-2379, 2010.

[25] Y. Fu and J. Zhang, "Modeling and analysis of microtubules based on a modified couple stress theory," Physica E, vol. 42, no. 5, pp. 1741-1745, 2010.

[26] Y. Gao and F. Lei, "Small scale effects on the mechanical behaviors of protein microtubules based on the nonlocal elasticity theory," Biochemical and Biophysical Research Communications, vol. 387, no. 3, pp. 467-471, 2009.

[27] A. Farajpour, A. Rastgoo, and M. Mohammadi, "Surface effects on the mechanical characteristics of microtubule networks in living cells," Mechanics Research Communications, vol. 57, pp. 18-26, 2014.

[28] H.-S. Shen, "Buckling and postbuckling of radially loaded microtubules by nonlocal shear deformable shell model," Journal of Theoretical Biology, vol. 264, no. 2, pp. 386-394, 2010.

[29] H.-S. Shen, "Application of nonlocal shell models to microtubule buckling in living cells," in Advances in Cell Mechanics, S. Li and B. Sun, Eds., pp. 257-316, Higher Education Press; Beijing, China, Springer, Heidelberg, Germany, 2011.

[30] H.-S. Shen, "Nonlocal shear deformable shell model for torsional buckling and postbuckling of microtubules in thermal environments," Mechanics Research Communications, vol. 54, pp. 83-95, 2013.

[31] A. Debbouche, D. Baleanu, and R. P. Agarwal, "Nonlocal nonlinear integrodifferential equations of fractional orders," Boundary Value Problems, vol. 2012, article 78, 2012.

[32] A. Debbouche and D. Baleanu, "Controllability of fractional evolution nonlocal impulsive quasilinear delay integrodifferential systems," Computers and Mathematics with Applications, vol. 62, no. 3, pp. 1442-1450, 2011.

[33] F. Daneshmand, E. Ghavanloo, and M. Amabili, "Wave propagation in protein microtubules modeled as orthotropic elastic shells including transverse shear deformations," Journal of Biomechanics, vol. 44, no. 10, pp. 1960-1966, 2011.

[34] K. M. Liew, P. Xiang, and Y. Sun, "A continuum mechanics framework and a constitutive model for predicting the orthotropic elastic properties of microtubules," Composite Structures, vol. 93, no. 7, pp. 1809-1818, 2011.

[35] X. Liu, Y. Zhou, H. Gao, and J. Wang, "Anomalous flexural behaviors of microtubules," Biophysical Journal, vol. 102, no. 8, pp. 1793-1803, 2012.

[36] C. P. Brangwynne, F. C. MacKintosh, S. Kumar et al., "Microtubules can bear enhanced compressive loads in living cells because of lateral reinforcement," Journal of Cell Biology, vol. 173, no. 5, pp. 733-741, 2006.

[37] M. Das, A. J. Levine, and F. C. MacKintosh, "Buckling and force propagation along intracellular microtubules," Europhysics Letters, vol. 84, no. 1, Article ID 18003, 2008.

[38] H. Jiang and J. Zhang, "Mechanics of microtubule buckling supported by cytoplasm," Journal of Applied Mechanics, Transactions ASME, vol. 75, no. 6, Article ID 061019, 9 pages, 2008.

[39] P. Xiang and K. M. Liew, "Free vibration analysis of microtubules based on an atomistic-continuum model," Journal of Sound and Vibration, vol. 331, no. 1, pp. 213-230, 2012.

[40] M. K. Zeverdejani and Y. T. Beni, “The nano scale vibration of protein microtubules based on modified strain gradient theory," Current Applied Physics, vol. 13, no. 8, pp. 1566-1576, 2013.
[41] T.-Z. Yang, S. Ji, X.-D. Yang, and B. Fang, "Microfluid-induced nonlinear free vibration of microtubes," International Journal of Engineering Science, vol. 76, pp. 47-55, 2014.

[42] S. Adali, "Variational principles for multi-walled carbon nanotubes undergoing buckling based on nonlocal elasticity theory," Physics Letters A, vol. 372, no. 35, pp. 5701-5705, 2008.

[43] S. Adali, "Variational principles for transversely vibrating multiwalled carbon nanotubes based on nonlocal euler-bernoulli beam model," Nano Letters, vol. 9, no. 5, pp. 1737-1741, 2009.

[44] S. Adali, "Variational principles for multi-walled carbon nanotubes undergoing nonlinear vibrations by semi-inverse method," Micro and Nano Letters, vol. 4, pp. 198-203, 2009.

[45] I. Kucuk, I. S. Sadek, and S. Adali, "Variational principles for multiwalled carbon nanotubes undergoing vibrations based on nonlocal Timoshenko beam theory," Journal of Nanomaterials, vol. 2010, Article ID 461252, 7 pages, 2010.

[46] S. Adali, "Variational principles for vibrating carbon nanotubes modeled as cylindrical shells based on strain gradient nonlocal theory," Journal of Computational and Theoretical Nanoscience, vol. 8, no. 10, pp. 1954-1962, 2011.

[47] C. Y. Ahn, "Robust myocardial motion tracking for echocardiography: variational framework integrating local-to-global deformation," Computational and Mathematical Methods in Medicine, vol. 2013, Article ID 974027, 14 pages, 2013.

[48] J. He, "Semi-inverse method of establishing generalized variational principles for fluid mechanics with emphasis on turbomachinery aerodynamics," International Journal of Turbo and Jet Engines, vol. 14, no. 1, pp. 23-28, 1997.

[49] J. He, "Variational principles for some nonlinear partial differential equations with variable coefficients," Chaos, Solitons and Fractals, vol. 19, no. 4, pp. 847-851, 2004.

[50] J.-H. He, "Variational approach to $(2+1)$-dimensional dispersive long water equations," Physics Letters A: General, Atomic and Solid State Physics, vol. 335, no. 2-3, pp. 182-184, 2005.

[51] J. He, "Variational principle for two-dimensional incompressible inviscid flow, Physics Letters A, vol. 371, no. 1-2, pp. 39-40, 2007.

[52] J.-H. He, "Lagrangian for nonlinear perturbed heat and wave equations," Applied Mathematics Letters, vol. 26, no. 1, pp. 158159, 2013.

[53] Z. Tao, "Variational approach to the inviscid compressible fluid," Acta Applicandae Mathematicae, vol. 100, no. 3, pp. 291-294, 2008.

[54] W. Zhang, "Generalized variational principle for long waterwave equation by He's semi-inverse method," Mathematical Problems in Engineering, vol. 2009, Article ID 925187, 5 pages, 2009.

[55] X.-W. Zhou and L. Wang, "A variational principle for coupled nonlinear Schrödinger equations with variable coefficients and high nonlinearity," Computers \& Mathematics with Applications, vol. 61, no. 8, pp. 2035-2038, 2011.

[56] Z. L. Tao and G. H. Chen, "Remark on a constrained variational principle for heat conduction,” Thermal Science, vol. 17, pp. 951952, 2013.

[57] J. He and E. W. M. Lee, "A constrained variational principle for heat conduction," Physics Letters A, vol. 373, no. 31, pp. 26142615, 2009.

[58] X.-W. Li, Y. Li, and J.-H. He, "On the semi-inverse method and variational principle," Thermal Science, vol. 17, pp. 1565-1568, 2013. 
[59] D.-D. Fei, F.-J. Liu, P. Wang, and H.-Y. Liu, "A short remark on He-Lee's variational principle for heat conduction," Thermal Science, vol. 17, pp. 1561-1563, 2013.

[60] Z.-B. Li and J. Liu, "Variational formulations for soliton equations arising in water transport in porous soils," Thermal Science, vol. 17, no. 5, pp. 1483-1485, 2013.

[61] J.-H. He, "Asymptotic methods for solitary solutions and compactons," Abstract and Applied Analysis, vol. 2012, Article ID 916793, 130 pages, 2012. 


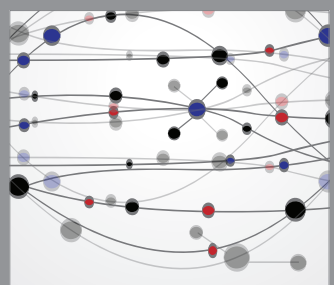

The Scientific World Journal
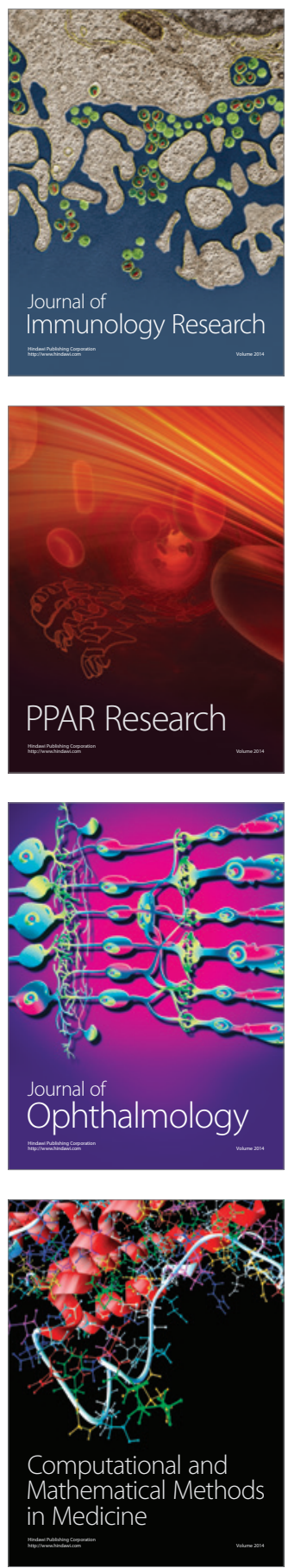

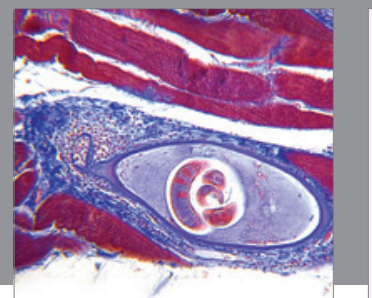

Gastroenterology

Research and Practice
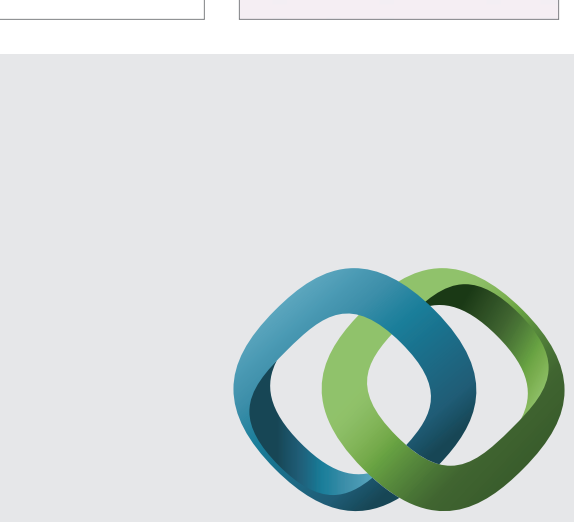

\section{Hindawi}

Submit your manuscripts at

http://www.hindawi.com
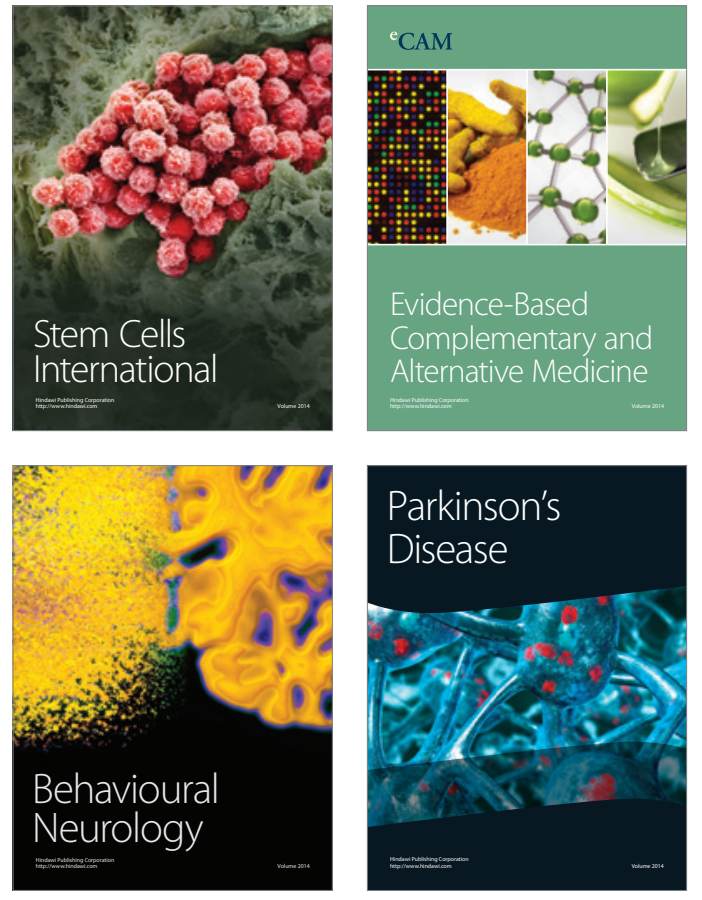
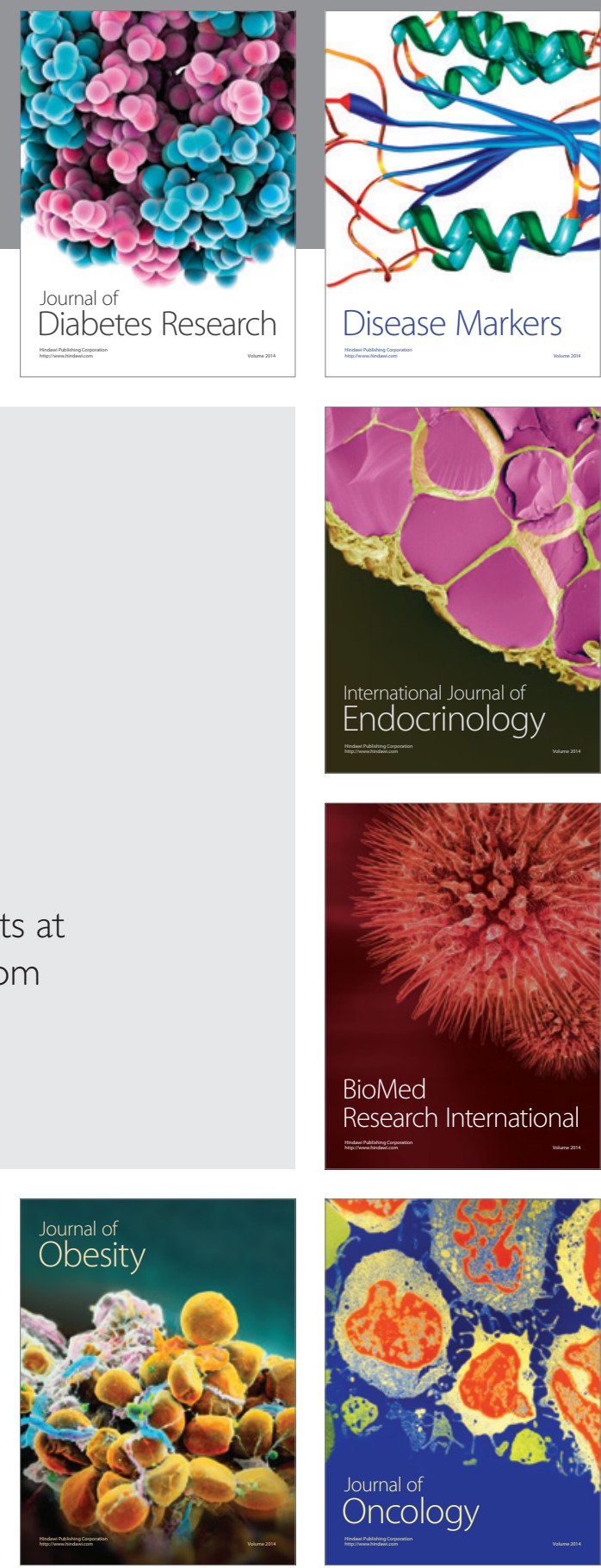

Disease Markers
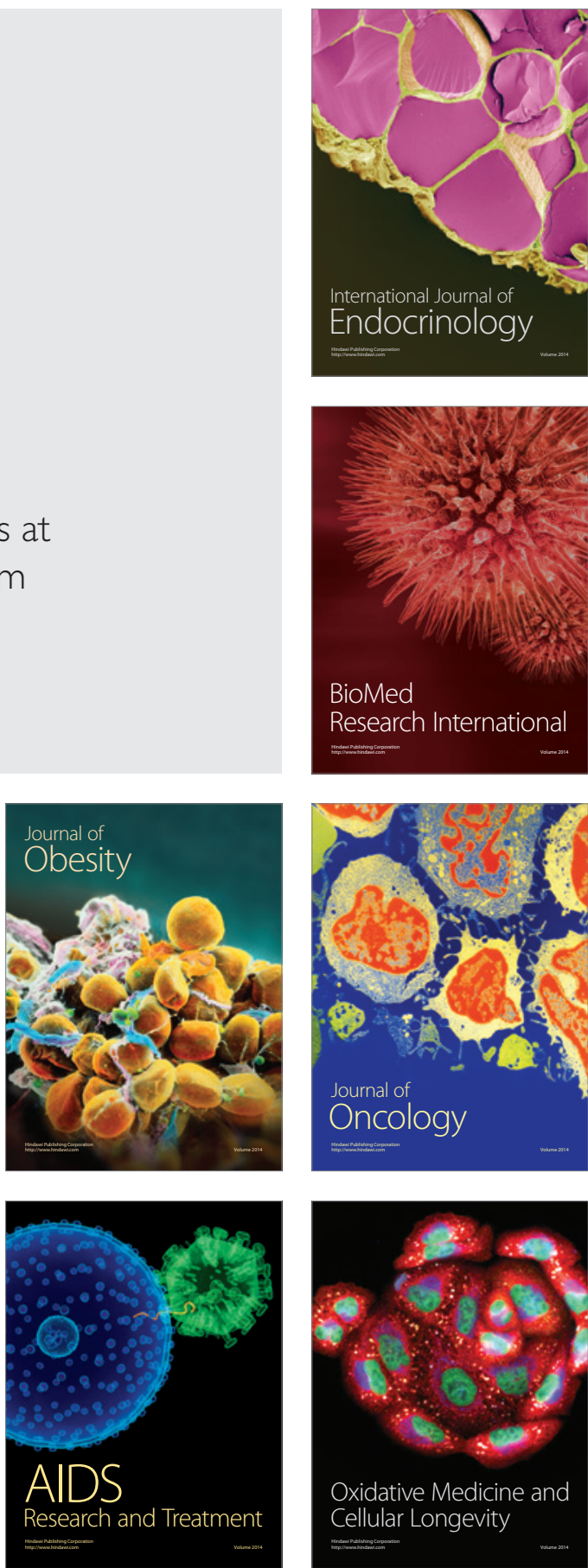